Marquette University

e-Publications@Marquette

Biomedical Engineering Faculty Research and

Publications

Biomedical Engineering, Department of

3-1-2005

\title{
Circumferential Vascular Deformation after Stent Implantation Alters Wall Shear Stress Evaluated with Time-Dependent 3D Computational Fluid Dynamics Models
}

\author{
John F. LaDisa \\ Marquette University, john.ladisa@marquette.edu \\ Lars E. Olson \\ Marquette University \\ Ismail Guler \\ Douglas Anthony Hettrick \\ Marquette University \\ Judy R. Kersten \\ Medical College of Wisconsin
}

See next page for additional authors

Follow this and additional works at: https://epublications.marquette.edu/bioengin_fac

Part of the Biomedical Engineering and Bioengineering Commons

\section{Recommended Citation}

LaDisa, John F.; Olson, Lars E.; Guler, Ismail; Hettrick, Douglas Anthony; Kersten, Judy R.; Warltier, David C.; and Pagel, Paul S., "Circumferential Vascular Deformation after Stent Implantation Alters Wall Shear Stress Evaluated with Time-Dependent 3D Computational Fluid Dynamics Models" (2005). Biomedical Engineering Faculty Research and Publications. 214.

https://epublications.marquette.edu/bioengin_fac/214 


\section{Authors}

John F. LaDisa, Lars E. Olson, Ismail Guler, Douglas Anthony Hettrick, Judy R. Kersten, David C. Warltier, and Paul S. Pagel

This article is available at e-Publications@Marquette: https://epublications.marquette.edu/bioengin_fac/214 
Marquette University

e-Publications@Marquette

\section{Biomedical Engineering Faculty Research and Publications/College of} Engineering

This paper is NOT THE PUBLISHED VERSION; but the author's final, peer-reviewed manuscript. The published version may be accessed by following the link in the citation below.

Journal of Applied Physiology, Vol. 98, No. 3 (March 2005): 947-957. DOI. This article is (c) American Physiological Society and permission has been granted for this version to appear in $\underline{\mathrm{e}}-$

Publications@Marquette. American Physiological Society does not grant permission for this article to be further copied/distributed or hosted elsewhere without the express permission from American Physiological Society.

\section{Circumferential Vascular Deformation After Stent Implantation Alters Wall Shear Stress Evaluated with Time-Dependent 3D Computational Fluid Dynamics Models}

John F. LaDisa Jr.

Departments of Anesthesiology, Department of Biomedical Engineering, Marquette University, Milwaukee, Wisconsin

Lars E. Olson

Department of Biomedical Engineering, Marquette University, Milwaukee, Wisconsin Ismail Guler

Boston Scientific, Maple Grove, Minnesota

Douglas A. Hettrick 
Departments of Anesthesiology, Department of Biomedical Engineering, Marquette University, Milwaukee, Wisconsin

\section{Judy R. Kersten}

Departments of Anesthesiology, Pharmacology and Toxicology, the Medical College of Wisconsin, Milwaukee, Wisconsin

\section{David C. Warltier}

Departments of Anesthesiology, Department of Biomedical Engineering, Marquette University, Milwaukee, Wisconsin

Medicine (Division of Cardiovascular Diseases), and Pharmacology and Toxicology, the Medical College of Wisconsin and the Clement J. Zablocki Veterans Affairs Medical Center, Milwaukee, Wisconsin

\section{Paul S. Pagel}

Departments of Anesthesiology, Department of Biomedical Engineering, Marquette University, Milwaukee, Wisconsin

\section{Abstract}

The success of vascular stents in the restoration of blood flow is limited by restenosis. Recent data generated from computational fluid dynamics (CFD) models suggest that stent geometry may cause local alterations in wall shear stress (WSS) that have been associated with neointimal hyperplasia and subsequent restenosis. However, previous CFD studies have ignored histological evidence of vascular straightening between circumferential stent struts. We tested the hypothesis that consideration of stent-induced vascular deformation may more accurately predict alterations in indexes of WSS that may subsequently account for histological findings after stenting. We further tested the hypothesis that the severity of these alterations in WSS varies with the degree of vascular deformation after implantation. Steady-state and time-dependent simulations of three-dimensional CFD arteries based on canine coronary artery measurements of diameter and blood flow were conducted, and WSS and WSS gradients were calculated. Circumferential straightening introduced areas of high WSS between stent struts that were absent in stented vessels of circular cross section. The area of vessel exposed to low WSS was dependent on the degree of circumferential vascular deformation and axial location within the stent. Stents with four vs. eight struts increased the intrastrut area of low WSS in vessels, regardless of cross-sectional geometry. Elevated WSS gradients were also observed between struts in vessels with polygonal cross sections. The results obtained using three-dimensional CFD models suggest that changes in vascular geometry after stent implantation are important determinants of WSS distributions that may be associated with subsequent neointimal hyperplasia.

stents restore blood flow through stenotic regions of the arterial vasculature. Unfortunately, restenosis after stent deployment remains a persistent problem. The mechanisms responsible for restenosis are not yet fully elucidated. Previous studies demonstrated that cell density gradients and neointimal hyperplasia were more likely to develop in regions exposed to nonuniform wall shear stress (WSS) $(19,20,22)$. We recently reported that the geometric properties and deployment ratio of an implanted stent influenced the amount of the vessel wall subjected to WSS values that have been implicated in neointimal hyperplasia using a three-dimensional (3D) computational fluid dynamics (CFD) model (16). Implicit in this previous investigation and other CFD studies is the assumption that the cross-sectional geometry of the artery remains entirely circular after the stent has been implanted. However, several previous histological studies from experimental animals and humans demonstrated that stent deployment produces circumferential straightening of vascular segments between adjacent stent struts in both normal and diseased vessels (Fig. 1) $(1,4,7,26)$. As a result of these in vivo data, we used steady-state and time-dependent 3D CFD models to test the hypothesis that circumferential arterial deformation after stent implantation imparts distinctive alterations in WSS that are not detected by using a 
standard circular model of vascular cross section. We further tested the hypothesis that the severity of vascular deformation within the stented segment increases the area of the computational vessel exposed to distributions of WSS implicated in the development of neointimal hyperplasia.

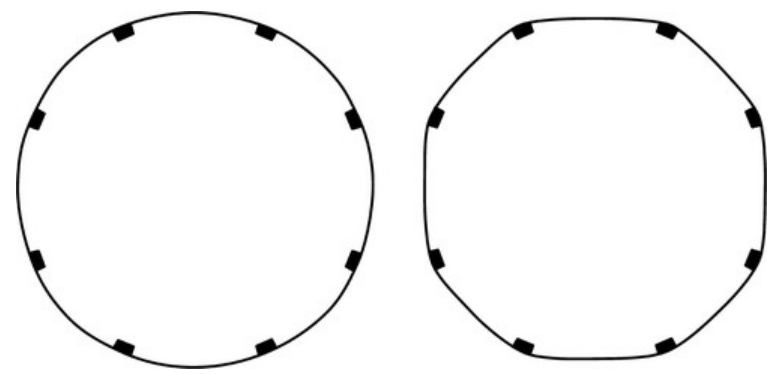

Fig. 1.Theoretical representations of normal arteries demonstrating circular cross section (left) or straightening of the artery between adjacent struts (right) after stent implantation.

\section{MATERIALS AND METHODS}

\section{Creation of theoretical stented arteries.}

An automated geometric construction and mesh generation algorithm custom designed by use of MATLAB (MathWorks, Natick, MA, www.mathworks.com) was used to create computational arteries containing a slottedtube stent embedded in a cylindrical artery, or arteries that conform to the struts of the implanted stent (see Fig. 9). CFD models were created from measurements of canine left anterior descending coronary artery blood flow and diameter, as described previously (14). Simulations were performed using four computational arteries composed of structured hexahedral control volumes arranged in a three-domain butterfly design that exploited symmetrical properties to model one-fourth of the vessel. Computational vessels were created using the stent-to-artery diameter convention of 1.2 to $1(7,31)$ and consisted of four or eight axial and circumferential repeating strut sections that differentially altered axial cross-sectional geometry (Fig. 9).

Simulation results from computational vessels that conformed to the geometry of the stent were compared with those in which a circular cross section was maintained after stent deployment (Table 1). The diameter of the native vessel for all simulations was $2.74 \mathrm{~mm}$. The thickness and width of stent struts were 0.096 and $0.197 \mathrm{~mm}$, respectively, in all simulations. The length of all computational stents was $16 \mathrm{~mm}$.

Table 1. Stent properties and indexes of wall shear stress

\begin{tabular}{|c|c|c|c|c|}
\hline Cross-sectional shape & Circular & Polygonal & Circular & Polygonal \\
\hline Number of struts & 4 & 4 & 8 & 8 \\
\hline Stent-to-artery ratio, \% & 4.43 & 4.43 & 8.83 & 8.83 \\
\hline Proximal intrastrut WSS $<5 \mathrm{dyn} / \mathrm{cm}^{2}, \mathrm{~mm}^{2}$ & 163.9 & 170.8 & 54.5 & 49.3 \\
\hline Middle intrastrut WSS $<5 \mathrm{dyn} / \mathrm{cm}^{2}, \mathrm{~mm}^{2}$ & 128.9 & 131.7 & 37.2 & 35.1 \\
\hline Distal intrastrut WSS $<5 \mathrm{dyn} / \mathrm{cm}^{2}, \mathrm{~mm}^{2}$ & 126.7 & 134.7 & 34.3 & 33.0 \\
\hline WSSG $>20 \mathrm{dyn} / \mathrm{cm}^{3}, \mathrm{~mm}^{2}$ & 76.8 & 100 & 76.4 & 89.2 \\
\hline Maximum WSSG, dyn/cm³ & 221 & 706 & 247 & 248 \\
\hline
\end{tabular}

WSS, wall shear stress; WSSG, wall shear stress gradient.

\section{Computational model simulations.}

Simulations were performed using the commercially available software package CFD-ACE (CFDRC, Huntsville, AL, www.cfdrc.com). This software uses a finite volume approach to solve the Navier-Stokes equations at the center of each hexahedral control volume. A steady-state velocity value corresponding to average blood flow 
velocity during one cardiac cycle in a canine coronary artery under resting conditions $(105 \mathrm{~mm} / \mathrm{s})$ was imposed as plug flow at the vessel inlet (14). Transient simulations were also conducted using a backward Euler temporal differencing method to investigate changes in WSS within the stented portion of vessels subjected to this coronary artery velocity waveform (Fig. 2). Additional length (AL) was added to all arteries to allow for fully developed flow using the equation $\mathrm{AL}=0.06 \cdot \operatorname{Re} \cdot d$, where $\mathrm{Re}$ is the Reynolds number and $d$ is the vessel inlet diameter (6). Computational simulations were conducted assuming incompressible flow of a Newtonian fluid with a density of $1,060 \mathrm{~kg} / \mathrm{m}^{3}$ and viscosity of $3.7 \mathrm{cP}(13)$.

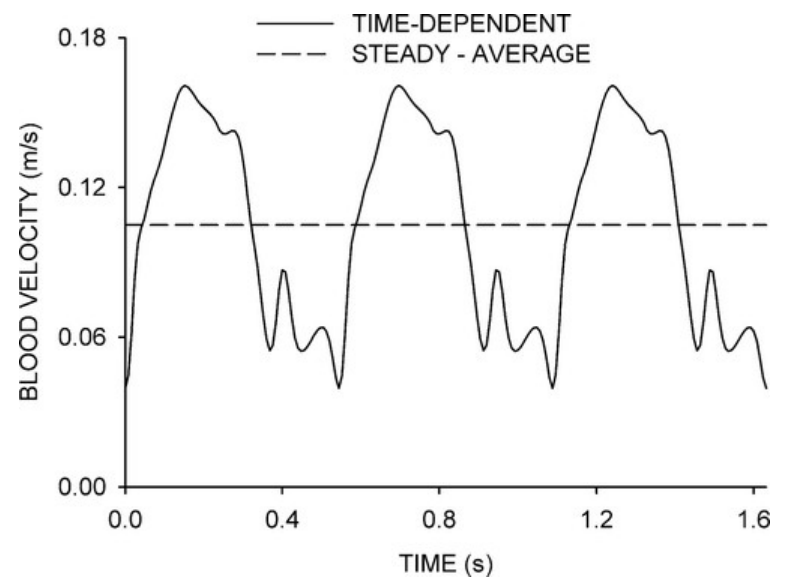

Fig. 2. Transient blood velocity waveform through the proximal portion of a canine left anterior descending coronary artery (14) used for the time-dependent simulations in the present investigation. The average blood velocity of $105 \mathrm{~mm} / \mathrm{s}$ depicted by the dashed line was also used as the inlet boundary condition for the steadystate (i.e., stationary) simulations.

\section{Determination of wall shear stress indexes.}

Wall shear stress was determined as the product of viscosity and shear rate. The CFD-ACE flow solver calculated shear rate using the strain rate tensor. Briefly, the viscous components of the stress tensor $\mathbf{T}$ were expressed as $\mathbf{T}=2 \mu \mathbf{D}$, where $\mu$ is the fluid viscosity and $\mathbf{D}$ is the strain rate tensor. $\mathbf{D}$ was expressed as $\mathbf{D}=1 / 2\left[(\nabla u)+(\nabla u)^{\top}\right]$, where $\nabla \mathbf{u}$ is the velocity gradient. During incompressible flow, the CFD-ACE flow solver calculates shear rate using the second invariant of the $\mathbf{D}$ tensor. Thus shear rate $(\gamma)$ was determined as $\gamma=\left(2 \mathbf{D}_{i j} \mathbf{D}_{i j}\right)^{1 / 2}$ where $\mathbf{D}_{i j} \mathbf{D}_{i j}$ is the inner product of the strain rate tensor with itself. Therefore,

$$
\gamma=\left\{2\left[\left(\frac{\partial u}{\partial x}\right)^{2}+\left(\frac{\partial v}{\partial y}\right)^{2}+\left(\frac{\partial w}{\partial z}\right)^{2}\right]+\left(\frac{\partial u}{\partial y}+\frac{\partial v}{\partial x}\right)^{2}+\left(\frac{\partial u}{\partial z}+\frac{\partial w}{\partial x}\right)^{2}+\left(\frac{\partial v}{\partial z}+\frac{\partial w}{\partial y}\right)^{2}\right\}^{1 / 2}
$$

where $u, v$, and $w$ are the $x, y$ and $z$ components of velocity vector, $u$, respectively.

This definition accounts for pure shear as well as extensional or elongational deformation in the flow domain.

Spatial wall shear stress gradients (WSSG) were also calculated by a previously described technique (9). WSSG was used to quantify the influence of nonuniform hemodynamic forces on adjacent intravascular cells and may correlate with the location of neointimal hyperplasia in vivo $(2,29,30)$. Briefly, a spatially identical 3D nodal shell of each computational vessel wall was produced by a modified version of the geometric construction and mesh generation algorithm discussed above. The Cartesian coordinates of the shell were converted into cylindrical coordinates, and the distribution of WSS obtained after simulation convergence was mapped onto the points of the new coordinate system by using MATLAB. A previous study suggested that spatial changes in frictional forces that act predominantly in the axial and circumferential directions were most likely to cause expansion of intracellular gaps and disrupt intracellular junctions (18). As a result, WSSG was calculated as 


$$
\left[\left(\frac{\partial \tau_{w, z}}{\partial z}\right)^{2}+\left(\frac{\partial \tau_{w, \theta}}{\partial \theta}\right)^{2}\right]^{1 / 2}
$$

where $\tau_{w, z}$ and $\tau_{w, \theta}$ are WSS in the axial and circumferential directions, respectively.

Previous studies conducted using an in vitro flow chamber and model of an end-to-side anastomosis have demonstrated that localized cellular proliferation may coincide with sudden pronounced changes in WSS during the cardiac cycle $(3,27,32)$. Therefore, temporal WSSG were calculated as $d \tau_{w} / d t$. Time-dependent alterations in WSS and temporal WSSG were determined at instrastrut points adjacent to the leading strut edge, at the center, and in the corner of strut units in the first proximal, middle, and last distal struts of the stent.

\section{Quantification of simulation results.}

The threshold for comparing distributions of low WSS between time-dependent simulations was established at 5 $\mathrm{dyn} / \mathrm{cm}^{2}$. Vascular regions subjected to WSS below this value have been shown to strongly correlate with sites of intimal thickening $(10,11)$. Additionally, near-wall velocity vectors were visualized at spatial locations in the proximal, middle, and distal portions of the stent to observe the behavior of blood flow in these regions.

Spatial WSSG correlate with sites of atherogenesis and have been used as an index of endothelial permeability that may represent an important initiating factor for the development of atherosclerosis (18). WSSG have also been used to examine the hypothesis that normally confluent cells react to nonuniform distributions of WSS in a way that promotes neointimal hyperplasia $(5,27,29)$. The percentage of the vessel wall subjected to WSSG values $>20 \mathrm{dyn} / \mathrm{cm}^{3}$ was quantified and compared between stationary simulations in the present investigation. The value of $20 \mathrm{dyn} / \mathrm{cm}^{3}$ was chosen because WSSG of this order of magnitude were generated in areas of neointimal hyperplasia within the toe region of an end-to-side arterial anastomosis in previous studies $(12,17,27)$. WSSG observed overlying stent struts were excluded from the analysis because these areas would not contain biologically active tissue immediately after implantation in vivo.

\section{RESULTS}

\section{Mesh independence.}

Steady-state simulations were performed on Silicon Graphics $025 \mathrm{k}$ workstations with $1 \mathrm{~Gb}$ of RAM that allowed for convergence within $72 \mathrm{~h}$. Twelve simulations were performed to optimize the stent parameters and investigate spatial mesh independence. Results were considered spatially independent of the computational mesh when differences in the distributions of WSS were $<6 \%$ between successive mesh densities $(13,25)$. Timedependent simulations were performed on a Dell Optiplex GX270 2.4-GHz workstation with 2 Gb of RAM that enabled convergence of simulations at a rate of $\sim 25$ time steps/day. Time step independence was investigated for the temporal simulations by subjecting computational vessels to the coronary artery blood flow velocity waveform illustrated in Fig. 2. Three permutations of this time-dependent coronary artery blood velocity waveform were obtained by interpolating from the measured waveform to contain 50,68, or 100 time points, resulting in time step increments of $10.9,8.0$, or $5.4 \mathrm{~ms}$. Three consecutive cardiac cycles were allowed to reach simulation convergence, and time step independence was then investigated by comparing distributions of WSS at equivalent points during each cardiac cycle and between waveform permutations. Figure 3 illustrates distributions of WSS along the luminal surface during peak diastole for each cardiac cycle. This figure demonstrates that a single cardiac cycle was sufficient to allow for the evolution of the time-varying initial conditions necessary for time step independence and an oscillatory steady-state solution. Similarly, Fig. 4 demonstrates how increasing the number of time steps beyond 68 points does not substantially alter temporal distributions of WSS within the stented region. 


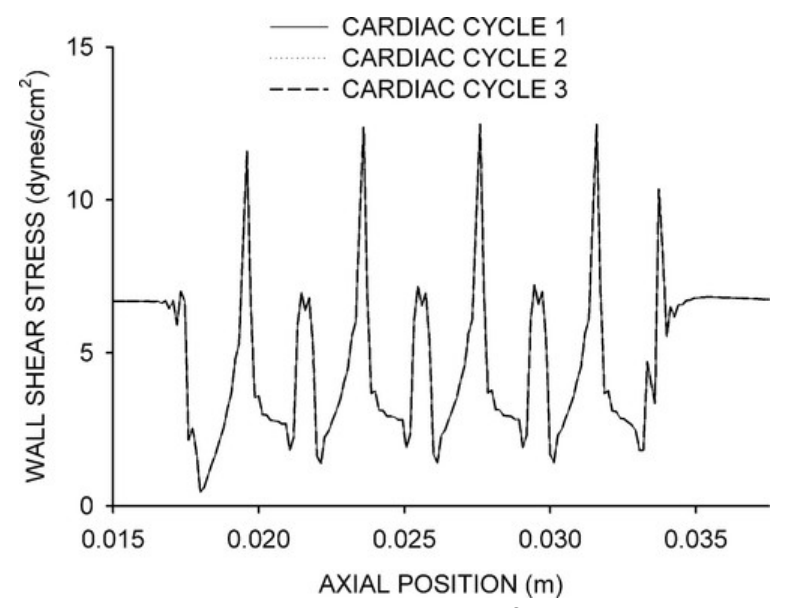

Fig. 3. Centerline distributions of wall shear stress (WSS) within a computational artery, demonstrating time step independence after 3 successive cardiac cycles.

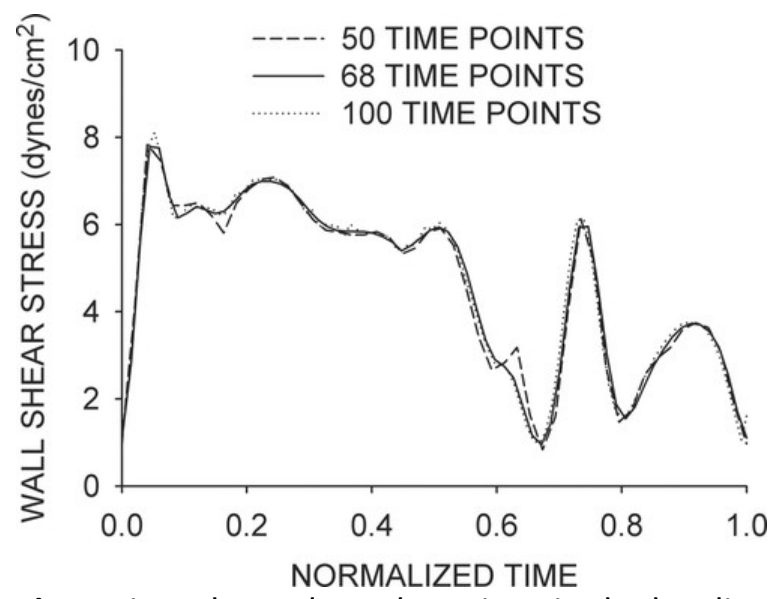

Fig. 4.Time-dependent alterations in the localized distribution of WSS at a point within the stented region for the transient coronary artery blood velocity waveform shown in Fig. 3, containing 50, 68, or 100 time points, resulting in time step increments of $10.9,8.0$, or $5.4 \mathrm{~ms}$. The time scale has been normalized to a single cardiac cycle. Pronounced undersampling of the local WSS waveform occurring around time 0.65 using a time step increment of $10.9 \mathrm{~ms}$ was sufficiently resolved by decreasing the time step size to $8.0 \mathrm{~ms}$.

\section{Steady-state simulations.}

Distributions of WSS for each CFD simulation are illustrated in Fig. 5. Lower WSS was observed within the stented region of all simulations, and stagnation zones occurred around stent struts. Circumferential straightening of the vessel wall between adjacent struts produced regions of high WSS within each repeating axial diamond of the stent that were not observed in the corresponding simulations conducted in vessels with circular cross sections. 


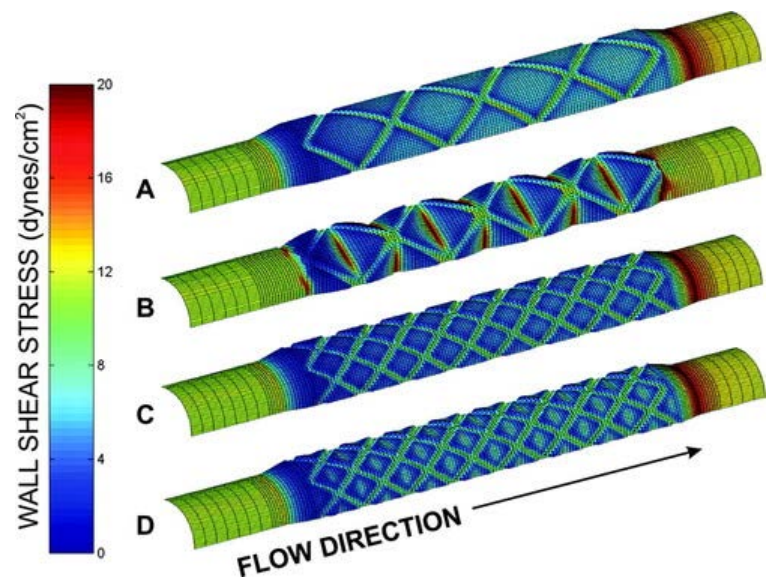

Fig. 5.Distributions of WSS throughout one-fourth of computational fluid dynamics (CFD) arteries with 4 $(A$ and $B)$ or $8(C$ and $D)$ struts in the presence of circular $(A$ and $C)$ or polygonal $(B$ and $D)$ cross sections. Although low WSS was observed in the localized stented region of all simulations, circumferential straightening of the vessel wall between adjacent struts produced regions of high WSS in simulations with polygonal cross sections that were not observed in the corresponding simulations conducted in vessels with circular cross sections.

Value-weighted near-wall velocity vectors in the first proximal, middle, and last distal repeating stent unit are demonstrated for each vessel in Fig. 6 . Reductions in the size of the vectors in the proximal compared with the middle region are caused by the sudden increase in luminal diameter within the stented region and account for the pronounced low WSS found in the proximal portion of the stent. The figure also demonstrates how adjacent blood layers converge before entering each repeating stent unit and diverge after entering each axial diamond. This divergence accounts for reductions in WSS adjacent to stent struts. Prolapse of the vessel into the flow domain also causes the flow imposed on the vessel wall to increase, resulting in higher WSS at the center of each repeating stent unit of the polygonal simulations. 

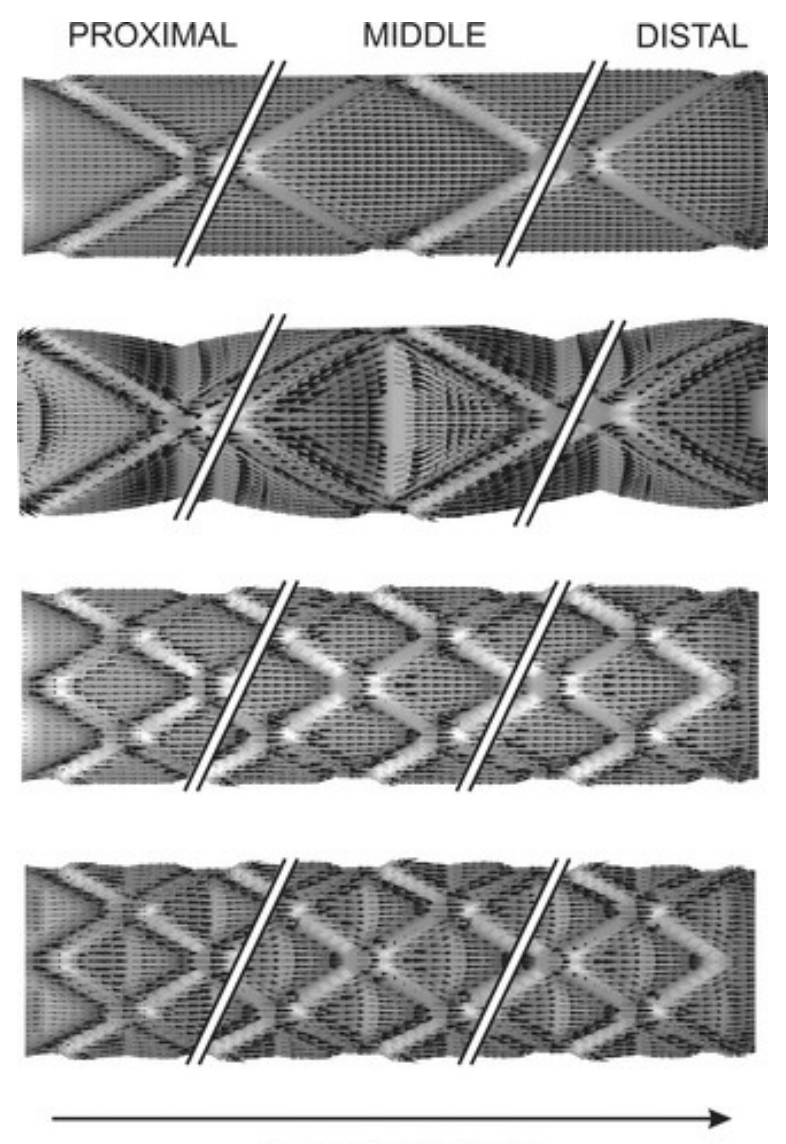

FLOW DIRECTION

Fig. 6. Value-weighted near-wall velocity vectors caused by localized increases or decreases in luminal diameter in the first proximal, middle, and most distal repeating stent unit of each computational vessel.

Plots of WSS vs. axial distance within the stented region and along the vessel centerline demonstrate that large wall deformations observed in the four-strut polygonal simulation produced increases in the amount of the vessel subjected to elevated WSS compared with the respective circular cross-sectional simulation, consistent with greater protrusion into the flow domain (Fig. 7). These large elevations in intrastrut WSS were attenuated when polygonal and circular simulations with eight stent struts were compared. 

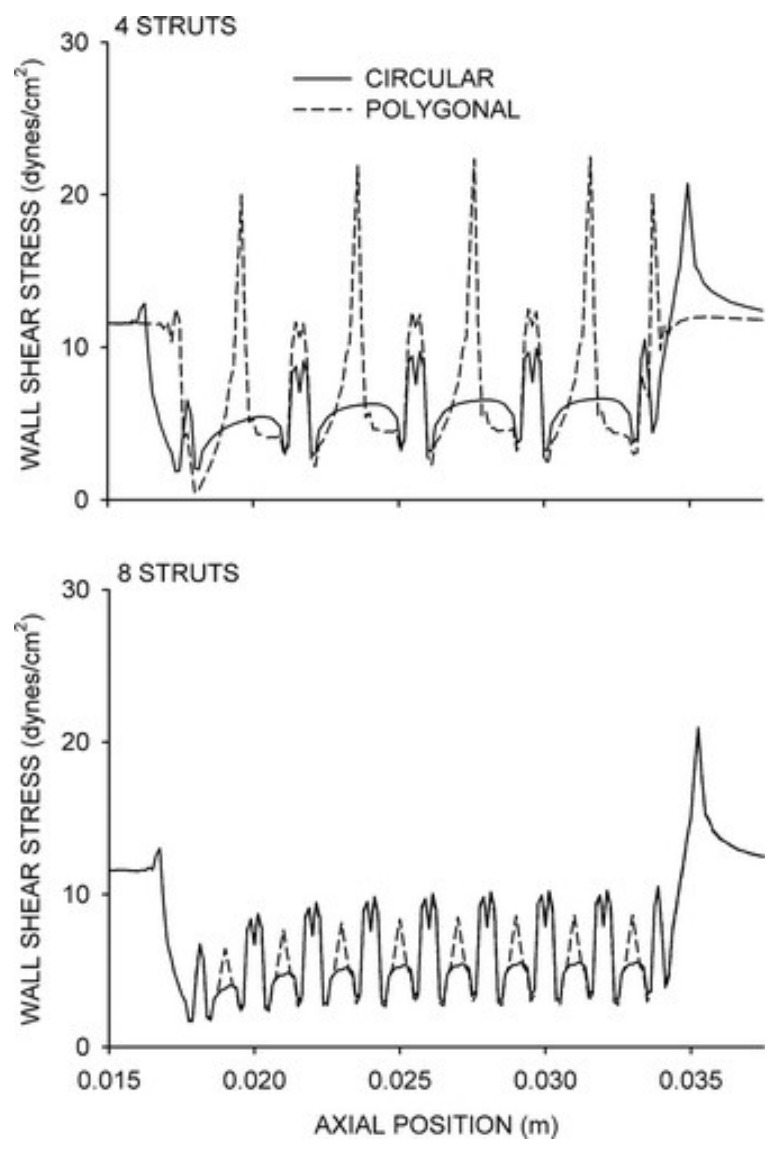

Fig. 7.Axial plots demonstrating the influence of stent-induced vascular prolapse on distributions of WSS. A: 4 struts; $B: 8$ struts. Vascular protrusion into the flow domain in simulations with polygonal cross sections produced increases in the amount of the vessel subjected to elevated WSS compared with the respective circular cross-sectional simulations.

Elevated spatial WSSG were noted at the stent inlet and outlet for each simulation (Fig. 8). A larger area of the computational vessel was subjected to WSSG $>20 \mathrm{dyn} / \mathrm{cm}^{3}$ in polygonal compared with circular simulations, independent of the number of struts (e.g., 100 vs. 76.8 for four-strut polygonal vs. circular stents, respectively; Table 1). In addition, the maximum WSSG observed in the four-strut polygonal simulated stent was markedly greater than that observed in the analogous circular stent (706 compared with $221 \mathrm{dyn} / \mathrm{cm}^{3}$, respectively). This stent geometry-induced difference in maximum WSSG did not occur in eight-strut stents. Analysis of the individual circumferential and axial components of WSSG indicated that the majority of WSSG elevations were caused by alterations in WSS in the axial (i.e., flow) direction (data not shown). 


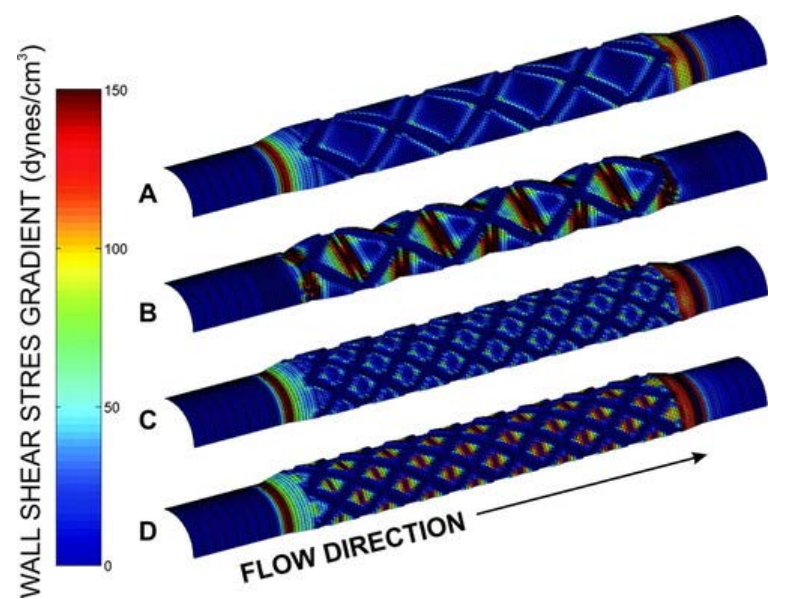

Fig. 8.Spatial wall shear stress gradients (WSSG) imparted on one-fourth of the computational vessel surface with $4(A$ and $B)$ or $8(C$ and $D)$ struts and circular $(A$ and $C)$ or polygonal $(B$ and $D)$ cross sections. Elevated spatial WSSG were noted at the stent inlet and outlet and adjacent to stent struts. Additionally, a greater amount of the computational vessel was subjected to elevated WSSG in polygonal compared with circular simulations.

\section{Time-dependent simulations.}

Time-dependent alterations in velocity profiles caused by flow acceleration and deceleration within each computational artery are illustrated in Fig. 9. Temporal deviations in the spatial distributions of WSS throughout the cardiac cycle resulting from modest differences in these velocity profiles are illustrated in Fig. 10.

Pronounced stagnation regions developed just before flow deceleration at the local and global maxima of the flow waveform (rows 2, 4, and 6) in vessels with polygonal compared with circular cross sections. These stagnation regions persisted for the duration of systole in polygonal vessels as a result of vessel prolapse into flow domain that maintains higher values of WSS within the center of each repeating strut unit.

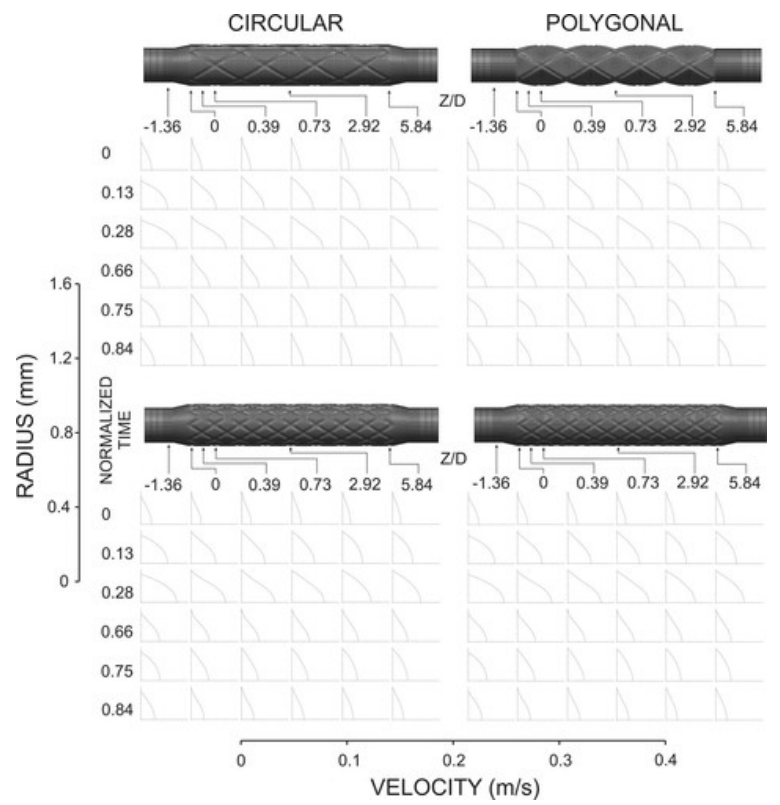

Fig. 9. Time-dependent alterations in velocity profiles caused by flow acceleration and deceleration within each computational artery. The velocity profiles are illustrated at several locations $(Z / D)$ with respect to the distal edge of the stent, where $Z$ is the axial location of the velocity profile and $D$ is the diameter of the native vessel in the unstented region. The scaling and labels for the $x$ - and $y$-axes (velocity and radius, respectively) have been enlarged for clarity on the bottom and left of the figure. 


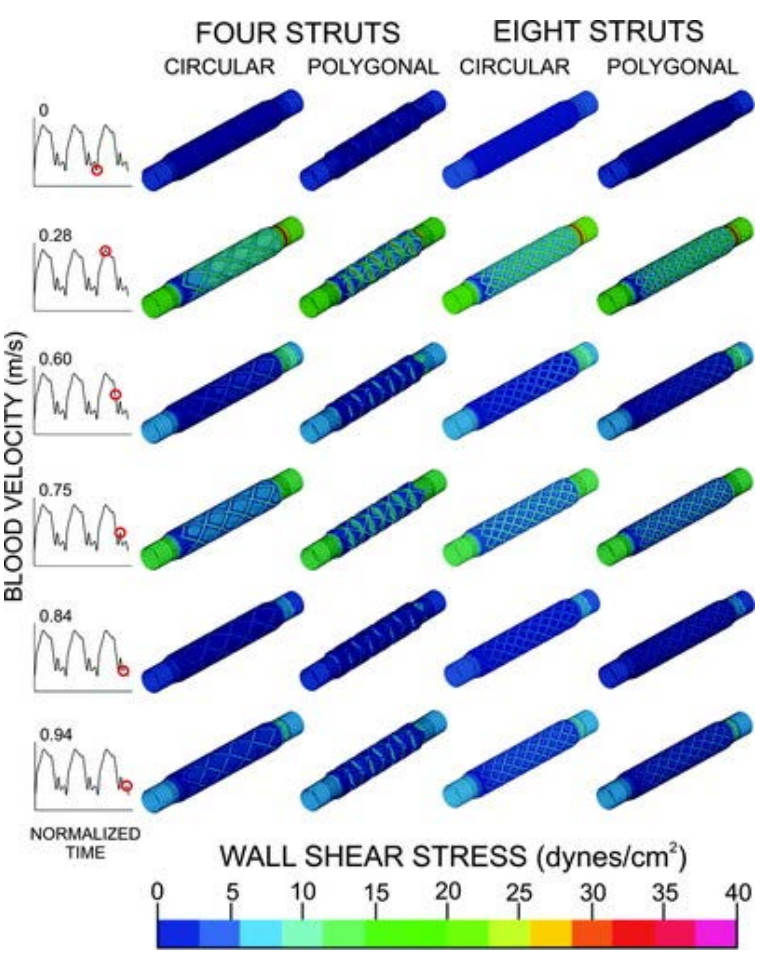

Fig. 10.Time-dependent alterations in spatial WSS throughout the cardiac cycle in the absence or presence of stent-induced vascular deformation caused by stents containing 4 or 8 struts.

The intrastrut area of low WSS was greatest within the region bounded by the first repeating proximal strut unit and progressively decreased in the middle and distal portions of the stent as flow attempted to reestablish after entering the stented portion of the vessel (Fig. 11). The vessel wall within the proximal, middle, and distal portions of the stent was subjected to low WSS for a greater percentage of the cardiac cycle in vessels containing four rather than eight stent struts. Additionally, the total intrastrut area of the vessel subjected to distributions of WSS $<5 \mathrm{dyn} / \mathrm{cm}^{2}$ during a single cardiac cycle was greater in four- vs. eight-strut vessels, regardless of axial location (Table 1 ). 


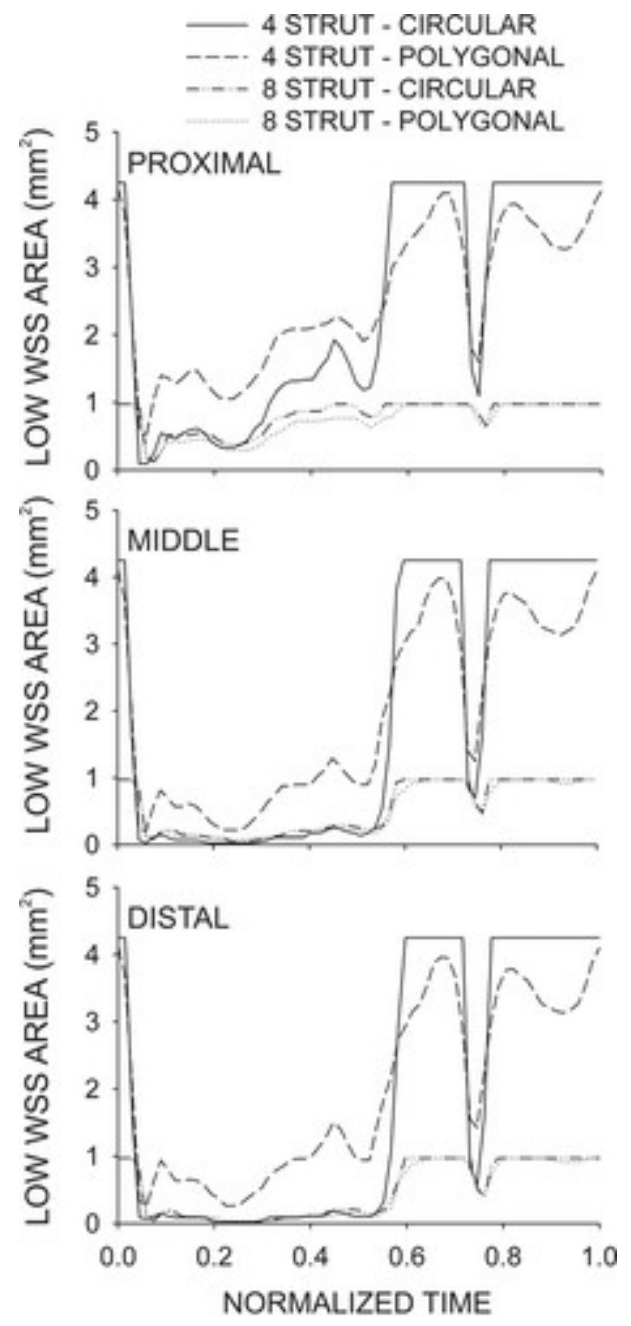

Fig. 11. Time-dependent alterations in the area of the vessel wall subjected to distributions of WSS $<5$ $\mathrm{dyn} / \mathrm{cm}^{2}$ within the first proximal, middle, and last distal repeating stent unit during a single cardiac cycle. The intrastrut area exposed to low WSS during times of increased blood flow (time 0.05-0.5) is greater in the proximal portion of the vessel, regardless of cross-sectional geometry, but is similar in the vascular sections of each vessel during periods of decreased blood flow.

No appreciable differences in temporal WSSG were observed adjacent to or in the corner of struts in the first proximal, middle, or last distal repeating strut unit, regardless of the number of struts or cross-sectional geometry (Fig. 12). Conversely, the luminal surface at the center of the struts in vessels with polygonal cross sections was subjected to temporal WSSG between 300 and $500 \mathrm{dyn} \cdot \mathrm{cm}^{-2} \cdot \mathrm{s}^{-1}$ shortly after the onset of systole and diastole. 


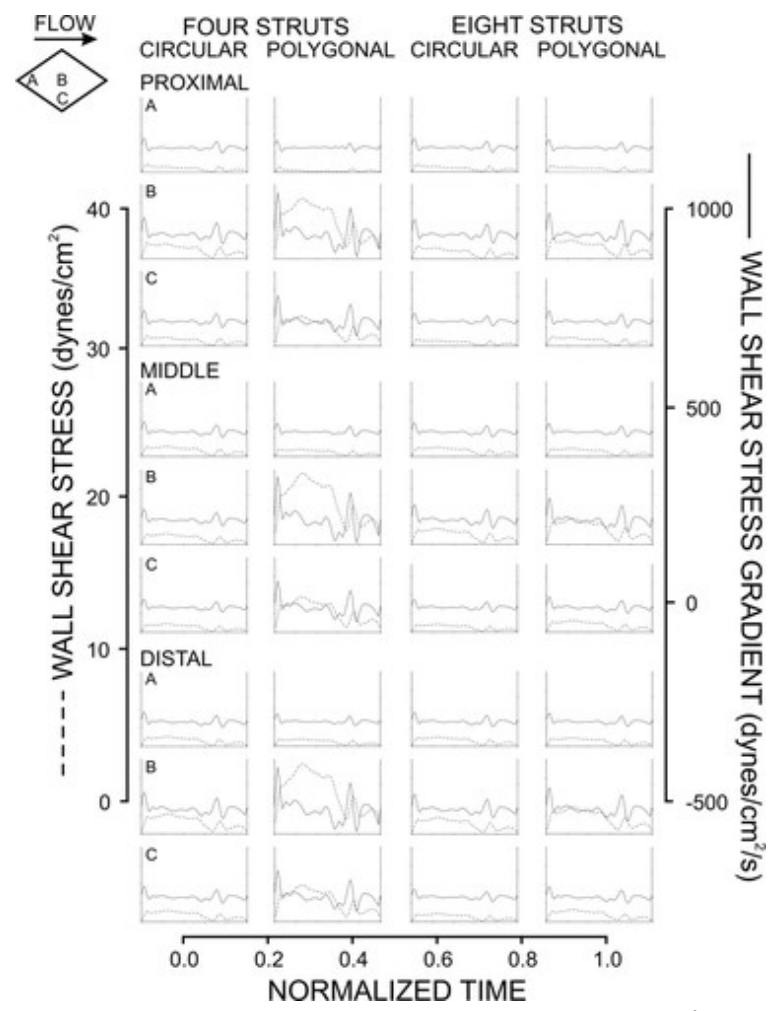

Fig. 12.Time-dependent alterations in WSS (dashed lines) and temporal WSSG (solid lines) observed adjacent to, in the corner, or at the center of struts (see inset in upper left corner) in the first proximal, middle, or last distal stent strut unit. Alterations in these indexes are illustrated in computational arteries with circular or polygonal vascular cross sections caused by stents containing 4 or 8 struts. The scaling and labels for the $x$ - and $y$-axes have been enlarged for clarity on the bottom, left, and right of the figure. Temporal WSSG observed adjacent to or in the corner of struts in the first proximal, middle, or last distal repeating strut unit were similar, regardless of the number of struts or cross-sectional geometry, but the luminal surfaces at the center of the struts in vessels with polygonal cross sections were subjected to potentially deleterious temporal WSSG between 300 and 500 dyn $\cdot \mathrm{cm}^{-2} \cdot \mathrm{s}^{-1}$ during portions of the cardiac cycle.

Time-dependent simulations were also used to investigate the potential error resulting from the assumption of imposing a steady-state velocity at the inlet of a vessel. Figure 13 illustrates distributions of WSS obtained from a steady-state inlet boundary condition of $105 \mathrm{~mm} / \mathrm{s}$ and the distributions of WSS resulting from the equivalent velocity value during flow acceleration toward peak diastole and flow deceleration. This analysis was performed using the computational vessel with four repeating strut units and polygonal vascular cross sections because this vessel results in the greatest amount of flow disturbances. The axial distributions of WSS reveal that the steadystate simulation underestimates distributions of WSS predicted during flow acceleration. Conversely, the steadystate solution overestimates distributions of shear stress along the vessel wall during flow deceleration. 


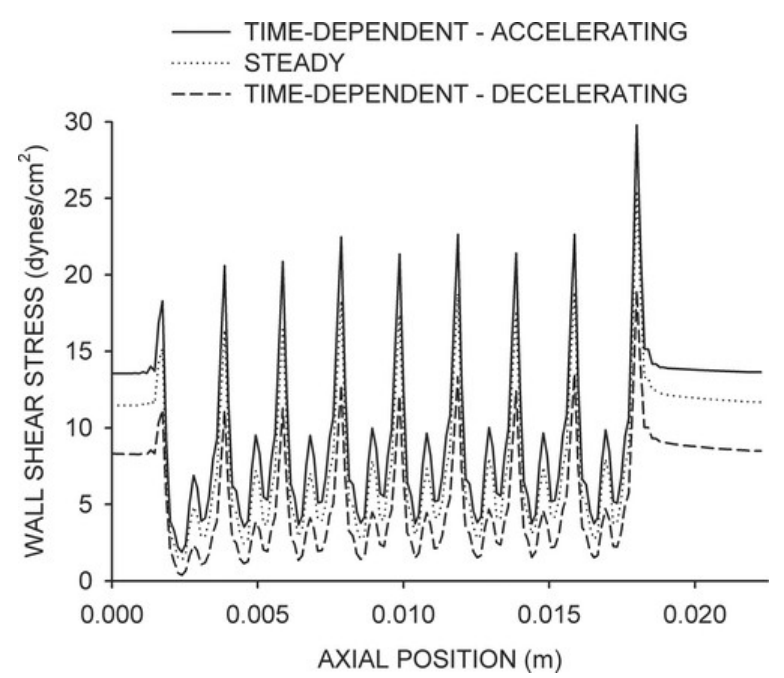

Fig. 13.Distributions of WSS corresponding to an inlet blood velocity of $105 \mathrm{~mm} / \mathrm{s}$ during a steady-state simulation or flow acceleration and deceleration in a time-dependent simulation. Steady-state simulations offer a reasonable approximation of the average distribution of WSS during the cardiac cycle at a fraction of the computational time, but time-dependent simulations that include the temporal term in the generalized transport equation provide more accurate distributions of WSS throughout the cardiac cycle.

\section{DISCUSSION}

Recent evidence indicates that alterations in WSS distributions after stent implantation may represent an important contributing factor in the process of restenosis. In particular, low WSS has been shown to influence the development of neointimal hyperplasia $(11,23)$. Nonuniform WSS within an arterial segment also affects the establishment of cell density gradients, gene expression, migration, and proliferation after vascular injury (1922). We have previously demonstrated that stents alter coronary artery WSS during maximum vasodilation in vivo (14). We have also shown that changes in WSS produced by stent implantation may be effectively modeled by 3D CFD and that design properties unique to the geometry of an implanted stent differentially influence the area of computational arteries subjected to indexes of WSS associated with vascular susceptibility to neointimal hyperplasia $(13,16)$. The present results using a 3D CFD model confirm and extend our previous findings and indicate that alterations in cross-sectional geometry after stent implantation exert an important impact on WSS distributions and WSSG. The results further indicate that polygonal computational vessels containing four struts are subjected to greater areas of low WSS and elevated WSSG than those with circular geometry and suggest that such an alteration in vascular shape may predispose the segment to subsequent neointimal hyperplasia. In contrast, these potentially deleterious alterations in low WSS distributions and high WSSG observed in four-strut polygonal vessels simulations did not occur in eight-strut polygonal models. It appears highly likely that this difference between four- and eight-strut polygonal simulations resulted because the eight-strut computational polygonal stent maintained a more circular profile and caused less disruption of the flow domain than the analogous four-strut simulation.

In contrast to the present findings, another study suggested that deviations from circular cross-sectional geometry after stenting may not necessarily be deleterious (26). These results indicated that implantation of a stent with six struts aligned to optimize axial flow exhibited relatively minor neointimal hyperplasia in the region between stent struts. Thus axial uniformity appears to be a critical factor in determining patency after stent implantation because alterations in the cross-sectional geometry along the length of the stented portion of the vessel introduce flow disturbances and minimize the overall area of the flow domain. This contention is supported by the present results demonstrating an increase in intrastrut area exposed to low WSS in vessels 
containing four vs. eight struts and those of a previous study demonstrating a greater amount of neointimal area in four compared with six strut stents 28 days after implantation in rabbit iliac arteries in vivo (7). Interestingly, the percentage of the intrastrut area subjected to low WSS in the present investigation was consistently greater for computational arteries containing eight vs. four struts when this area of low WSS was normalized by the luminal surface area bounded by this region. This finding demonstrates that increasing the stent-to-artery ratio may protect the luminal surface from potentially deleterious indexes of WSS. The present results with timedependent simulations further suggest that the vessel wall within the proximal portion of the stent is subjected to low WSS for a greater duration of the cardiac cycle than that near the middle or distal portions of stents. This suggests that neointimal hyperplasia may be greater at the entrance of the stent and is consistent with our preliminary histological findings in an experimental model of stent implantation in normal arteries in vivo (15).

The present results suggest that previously conducted CFD studies that assumed purely circular vascular geometry between struts may have important limitations because the amount of the vessel exposed to low WSS may be underestimated. The present results further suggest that such an assumption of circular geometry also underestimates the relative area of the segment subjected to elevated WSSG between struts. Importantly, our findings indicate that errors in WSS associated with the assumption of circumferential vascular cross sections after stent implantation may be substantial and depend on the geometry and number of struts contained in the stent that is implanted and modeled by CFD.

Profoundly elevated values of WSSG were observed within the center of each axial diamond of the stented segment in simulations with four struts. These values of WSSG were much greater than those previously reported within vascular segments after luminal intervention (17). Nevertheless, a previous study demonstrated that neointimal hyperplasia appeared to be greatest at the center of each axial diamond using a similar stent geometry in vivo (7). However, the present results demonstrate that these regions were also exposed to high WSS that should theoretically protect the vessel from neointimal hyperplasia. The results also suggest that markedly elevated WSSG in this region may provide a more pronounced stimulus to cellular proliferation that may counterbalance the relative protective effect of high WSS. Alternatively, localized temporal WSSG, as demonstrated in the present investigation, or endothelial and smooth muscle cell damage to the vessel wall during stent deployment, resulting from angioplasty balloon penetration through the stent struts, may also account for previously described neointimal hyperplasia in this region.

Previous studies have demonstrated that pronounced increases or decreases in temporal WSSG are localized to regions of neointimal hyperplasia along the bed of an arterial anastomosis (27). Temporal WSSG of 300 dyn $\cdot \mathrm{cm}^{-2} \cdot \mathrm{s}^{-1}$ have also been shown to cause endothelial cell proliferation in the absence of spatial WSSG by use of a parallel-plate flow chamber (32). Temporal WSSG results from the present investigation suggest that the shape of the vascular scaffolding created by the stent may subject localized intrastrut regions to temporal WSSG that may be associated with cellular proliferation. Moreover, low temporal WSSG adjacent to and in the corners of each repeating strut unit suggest that low WSS, not temporal WSSG, may predominate and be responsible for neointimal hyperplasia forming in these regions.

To the authors' knowledge, the present investigation represents the first time-dependent 3D CFD model describing alterations in localized WSS within a theoretical stented artery. Previous studies have discussed the disparity obtained between steady-state and time-dependent simulation results obtained in other vascular beds (25). The present results indicate that conclusions regarding distributions of WSS in stented arteries depend on the point of the cardiac cycle that is modeled by a steady-state inlet boundary condition. Figure 11 demonstrates that the intrastrut area exposed to low WSS during times of increased blood flow (time 0.050.5 ) is greater in the proximal portion of the vessel, regardless of cross-sectional geometry, but is similar in the vascular sections of each vessel during periods of decreased blood flow. Therefore, steady-state simulations using a mean or maximum blood flow velocity value may be more likely to elucidate potentially deleterious 
alterations in indexes of WSS, resulting from the geometry of the stent that is computationally modeled. Blood flow patterns and trends were similar between the distributions of WSS obtained from a steady-state inlet boundary condition corresponding to the average blood velocity throughout the cardiac cycle and those resulting from the equivalent time point during the time-dependent simulations (Figs. 5 and 10, respectively). The present results demonstrate that steady-state simulations can provide a reasonable estimate of the average distribution of shear stress imparted on the wall of stented vessels during a single cardiac cycle at approximately one-tenth of the computational time (Fig. 13). However, the present results also emphasize the importance of including the transient term in the generalized transport equation utilized by the flow solver. Full-scale timedependent simulations offer the benefit of visualizing temporal alterations in indexes of WSS resulting from flow acceleration or deceleration that are not expressed in quasi-steady or steady-state results.

The present results should be interpreted within the constraints of several potential limitations. The present simulation results were obtained by comparing normal arteries with circular or polygonal cross sections, but the geometry of atherosclerotic lesions after stent implantation may vary based on lesion composition and may also limit vascular concentricity $(8,24,28)$. A previous investigation demonstrated that implantation of $16-\mathrm{mm}$ slotted-tube stents into canine epicardial coronary arteries reduced compliance to zero in the region of the stent (14). However, localized intrastrut compliance may not be eliminated after implantation. To model this phenomenon would require simulations using fluid-structure interactions that are not yet ubiquitous in blood flow modeling applications. Compliance of the vascular wall in response increases in blood flow throughout the cardiac cycle may result in distributions of WSS that differ from those predicted by the present model in the proximal and distal stent-to-artery transitions regions. The disparity between distributions of WSS resulting from a compliant computational model and that predicted in the present investigation would likely be less pronounced in localized intrastrut regions as a result of the rigidity created by the implanted stent.

In summary, the present results using 3D CFD modeling indicate that polygonal cross-sectional vascular geometry after stent implantation may be associated with increases in the area of the vessel subjected to low WSS and high WSSG, factors that have been correlated with subsequent neointimal hyperplasia in vivo. Increasing the number of strut intersections produces a more circular geometry and reduces the amount of the simulated artery exposed to elevated spatial and temporal WSSG and the intrastrut area of potentially adverse distributions of WSS. Blood flow patterns were similar between distributions of WSS obtained from a steadystate inlet boundary condition corresponding to the average blood velocity during a cardiac cycle and those resulting from the equivalent time point in time-dependent simulations. However, time-dependent simulations accounted for flow acceleration and deceleration and suggested that steady-state simulations using a mean or maximum blood flow velocity value are more likely to elucidate potentially deleterious alterations in indexes of WSS resulting from the geometry of an implanted stent. The results also indicate that circumferential deformation used in this polygonal model of artery geometry appears to more accurately reflect acute changes in vascular conformation and WSS after stent implantation in vivo.

\section{GRANTS}

This work was supported in part by National Institutes of Health Grants HL-03690 (to Dr. Kersten), HL-63705 (to J. R. Kersten), HL-54820 (to D. C. Warltier), and GM-08377 (to D. C. Warltier) from the United States Public Health Service, Bethesda, MD.

\section{FOOTNOTES}

- The costs of publication of this article were defrayed in part by the payment of page charges. The article must therefore be hereby marked "advertisement" in accordance with 18 U.S.C. Section 1734 solely to indicate this fact. 
The authors recognize David A. Schwabe and John P. Tessmer for experimental support, Mary Lorence-Hanke for assistance in the preparation of this manuscript (Department of Anesthesiology, Medical College of Wisconsin), and Said Audi and Kristina Ropella in the Department of Biomedical Engineering at Marquette University for advice and support.

\section{AUTHOR NOTES}

- $\quad$ Address for reprint requests and other correspondence: P. S. Pagel, Medical College of Wisconsin, MEBM4280, 8701 Watertown Plank Rd., Milwaukee, WI 53226 (E-mail: pspagel@mcw.edu)

\section{REFERENCES}

1 Danenberg HD, Welt FG, Walker M, Seifert P, Toegel GS, and Edelman ER. Systemic inflammation induced by lipopolysaccharide increases neointimal formation after balloon and stent injury in rabbits. Circulation 105: 2917-2922, 2002.

2 DePaola N, Gimbrone MA Jr, Davies PF, and Dewey CF. Vascular endothelium responds to fluid shear stress gradients. Arterioscler Thromb 12: 1254-1257, 1992.

3 Ethier CR, Steinman DA, Zhang X, Karpik SR, and Ojha M. Flow waveform effects on end-to-side anastomotic flow patterns. J Biomech 31: 609-617, 1998.

4 Farb A, Weber DK, Kolodgie FD, Burke AP, and Virmani R. Morphological predictors of restenosis after coronary stenting in humans. Circulation 105: 2974-2980, 2002.

5 Finol EA and Amon CH. Blood flow in abdominal aortic aneurysms: pulsatile flow hemodynamics. $J$ Biomech Eng 123: 474-484, 2001.

6 Fox RW and McDonald AT. Introduction to Fluid Mechanics (4th ed.). New York: Wiley, 1992, p. 322.

7 Garasic JM, Edelman ER, Squire JC, Seifert P, Williams MS, and Rogers C. Stent and artery geometry determine intimal thickening independent of arterial injury. Circulation 101: 812-818, 2000.

8 Grewe PH, Deneke T, Machraoui A, Barmeyer J, and Muller KM. Acute and chronic tissue response to coronary stent implantation: pathologic findings in human specimen. J Am Coll Cardiol 35: 157163, 2000.

9 Kleinstreuer C, Hyun S, Buchanan JR Jr, Longest PW, Archie JP Jr, and Truskey GA. Hemodynamic parameters and early intimal thickening in branching blood vessels. Crit Rev Biomed Eng 29: 24-27, 2001.

10 Ku DN. Blood flow in arteries. Ann Rev Fluid Mech 29: 399-434, 1997.

11 Ku DN, Giddens DP, Zarins CK, and Glagov S. Pulsatile flow and atherosclerosis in the human carotid bifurcation. Positive correlation between plaque location and low oscillating shear stress. Arteriosclerosis 5: 293-302, 1985.

12 Kute SM and Vorp DA. The effect of proximal artery flow on the hemodynamics at the distal anastomosis of a vascular bypass graft: computational study. J Biomech Eng 123: 277-283, 2001.

13 LaDisa JF Jr, Guler I, Olson LE, Hettrick DA, Kersten JR, Warltier DC, and Pagel PS. Three-dimensional computational fluid dynamics modeling of alterations in coronary wall shear stress produced by stent implantation. Ann Biomed Eng 31: 972-980, 2003.

14 LaDisa JF Jr, Hettrick DA, Olson LE, Guler I, Gross ER, Kress TT, Kersten JR, Warltier DC, and Pagel PS. Coronary stent implantation alters coronary artery hemodynamics and wall shear stress during maximal vasodilation. J Appl Physiol 93: 1939-1946, 2002.

15 LaDisa JF Jr, Meier HT, Olson LE, Kersten JR, Warltier DC, and Pagel PS. Antegrade iliac artery stent implantation for the temporal and spatial examination of stent-induced neointimal hyperplasia and alterations in regional fluid dynamics. J Pharmacol Toxicol Meth Epub Sept 11, 2004.

16 LaDisa JF, Olson LE, Guler I, Hettrick DA, Audi SH, Kersten JR, Warltier DC, and Pagel PS. Stent design properties and deployment ratio influence indexes of wall shear stress: a three-dimensional computational fluid dynamics investigation within a normal artery. J Appl Physiol 97: 424-430, 2004. 
17 Lei M, Kleinstreuer C, and Archie JP Jr. Geometric design improvements for femoral graft-artery junctions mitigating restenosis. J Biomech 29: 1605-1614, 1996.

18 Lei M, Kleinstreuer C, and Truskey GA. A focal stress gradient-dependent mass transfer mechanism for atherogenesis in branching arteries. Med Eng Phys 18: 326-332, 1996.

19 Liu SQ and Goldman J. Role of blood shear stress in the regulation of vascular smooth muscle cell migration. IEEE Trans Biomed Eng 48: 474-483, 2001.

20 Liu SQ, Tang D, Tieche C, and Alkema PK. Pattern formation of vascular smooth muscle cells subjected to nonuniform fluid shear stress: mediation by the gradient of cell density. Am J Physiol Heart Circ Physiol 285: H1072-H1080, 2003.

21 Liu SQ, Tieche C, Dalin T, and Alkema P. Pattern formation of vascular smooth muscle cells subject to nonuniform fluid shear stress: role of platelet-derived growth factor $\beta$-receptor and Src. Am J Physiol Heart Circ Physiol 285: H1081-H1090, 2003.

22 Liu SQ, Zhong L, and Goldman J. Control of the shape of a thrombus-neointima-like structure by blood shear stress. J Biomech Eng 124: 30-36, 2002.

23 Moore JE Jr, Xu C, Glagov S, Zarins CK, and Ku DN. Fluid wall shear stress measurements in a model of the human abdominal aorta: oscillatory behavior and relationship to atherosclerosis. Atherosclerosis 110: 225-240, 1994.

24 Murata T, Hiro T, Fujii T, Yasumoto K, Murashige A, Kohno M, Yamada J, Miura T, and Matsuzaki M. Impact of the cross-sectional geometry of the post-deployment coronary stent on in-stent neointimal hyperplasia: an intravascular ultrasound study. Circ J 66: 489-493, 2002.

25 Myers JG, Moore JA, Ojha M, Johnston KW, and Ethier CR. Factors influencing blood flow patterns in the human right coronary artery. Ann Biomed Eng 29: 109-120, 2001.

26 Newman VS, Berry JL, Routh WD, Ferrario CM, and Dean RH. Effects of vascular stent surface area and hemodynamics on intimal thickening. J Vasc Interv Radiol 7: 387-393, 1996.

27 Ojha M. Spatial and temporal variations of wall shear stress within an end-to-side arterial anastomosis model. J Biomech 26: 1377-1388, 1993.

28 Schulz C, Herrmann RA, Beilharz C, Pasquantonio J, and Alt E. Coronary stent symmetry and vascular injury determine experimental restenosis. Heart 83: 462-467, 2000.

29 Tada S and Tarbell JM. Flow through internal elastic lamina affects shear stress on smooth muscle cells (3D simulations). Am J Physiol Heart Circ Physiol 282: H576-H584, 2002.

30 Tardy Y, Resnick N, Nagel T, Gimbrone MA, and Dewey CF. Shear stress gradients remodel endothelial monolayers in vitro via a cell proliferation-migration-loss cycle. Arterioscler Thromb Vasc Biol 17: 31023106, 1997.

31 Van Belle E, Tio FO, Couffinhal T, Maillard L, Pesseri J, and Isner JM. Stent endothelialization: time course, impact of local catheter delivery, feasibility of recombinant protein administration, and response to cytokine expedition. Circulation 95: 438-448, 1997.

32 White CR, Haidekker M, Bao X, and Frangos JA. Temporal gradients in shear, but not spatial gradients, stimulate endothelial cell proliferation. Circulation 103: 2508-2513, 2001. 